\title{
Sujeto y acción en la sociedad y en la historia
}

\author{
Miguel Ángel García Sánchez* \\ Centro de Estudios Históricos, CSIC
}

\section{Subject and action in Society and History}

\begin{abstract}
RESUMEN ABSTRACT
Participando de un esquema analítico que combina sociología e historia, el presente artículo aborda, en primer lugar, el concepto de acción, para poder entender a partir del mismo el papel desempeñado por el individuo dentro de los procesos sociales. En segundo lugar, se recupera la polémica desatada entre L. Althusser y $E$. $P$. Thompson en la década de los años 70 respecto al significado del sujeto dentro del método historiográfico. Se procede después a reflexionar acerca de la relación existente entre actor, grupo y estructura, recurriendo para ello a distintos científicos sociales, como A. Giddens o P. Bourdieu. Por último, se presentan propuestas recientes lanzadas por sociólogos (teoría de redes, intercambio social, cohesión) e historiadores (estrategias) que no pueden ser debidamente comprendidas sin conocer los antecedentes teóricos que se explican en los tres primeros apartados. Tal

Using a method which combines sociology and history, this paper deals firstly with the definition of the concept of action, as a means to understand the role played by the actor in society. Secondly, the debate between L. Althusser and E. P. Thompson is sketched in order to stress the importance attached to the actor within the historical method. Thirdly, I aim to look epistemologically at the relation between actor, group and structure, which has been explored by some social scientists, like $A$. Giddens or P. Bourdieu. Finally, I will be involved with some recent analytical proposals that sociologists (network, exchange, cohesion theories) and historians (strategies) have proposed, and that cannot be properly grasped without taking into account the theoretical background explained in the first three sections. This contextualization of the problem entails the main purpose of this paper.
\end{abstract}

* Becario FPU en el Departamento de Historia Moderna del Consejo Superior de Investigaciones Científicas e Investigador invitado en el Centre for Metropolitan History del Institute of Historical Research de la University of London. Este artículo se enmarca en las actividades que se desarrollan dentro del proyecto de investigación titulado «Cervantes y su época: teoría y práctica de la comunicación científica» (HUM 2004-04713/HIST). Ha sido financiado por el Ministerio de Educación y Ciencia y la Comunidad de Madrid y su investigador principal es el Dr. Alfredo Alvar Ezquerra. 
contextualización del problema constituye

el principal objetivo de estas páginas.

PALABRAS CLAVE:

Sujeto histórico / estructura / redes

sociales / estrategias.
KEYWORDS:

Historical actor / structure / social

networks / strategies.

\section{INTRODUCCIÓN}

Cuando leemos trabajos de F. Braudel o E. H. Hobsbawm, de E. Durkheim o R. Dahrendorf, descubrimos cómo la pregunta en torno a qué fuerza prima en los procesos sociales, si el sujeto o la estructura, está ahí presente, subyaciendo por debajo de las reflexiones de historiadores y sociólogos. En definitiva, profesionales de ambos campos parecen coincidir en que es el individuo quien dirige su porvenir por medio de las acciones que promueve, si bien no menos unánimes se muestran al reconocer que las comunidades humanas son algo más que un mero agregado de unidades independientes y que cuentan asimismo con capacidades propias para moldear las vidas de aquellos que las componen.

Ante esta realidad, tradicionalmente el historiador se ha afanado en buscar los porqués de aquellos hechos que observa en los documentos, no siempre consciente de que tal ejercicio es un arma de doble filo que bien puede dar lugar a un correcto análisis del que se extraigan conclusiones satisfactorias, bien puede desembocar en una construcción determinista obsesionada por hallar una causa última y definitiva ${ }^{1}$. Nunca olvidará, sin embargo, insertar en su trabajo la perspectiva del cambio, ese mecanismo por el cual la constatación de un hecho para un punto concreto en el tiempo varía respecto a otra realizada poco después, invitando tal ejercicio a la búsqueda de una razón que explique la mutación acaecida. Por el contrario, y con el objeto de ofrecer una introducción general a este artículo, el sociólogo ha procurado más bien elaborar modelos teóricos, basados en la observación empírica, para los cuales la cuestión de por qué se cambia se ve desplazada por la de cómo se funciona².

Puede parecer que con estas apreciaciones se están resucitando viejos debates hoy ya superados. En buena medida, por cierto, el propósito que se persigue con el presente artículo consiste en sintetizar algunas propuestas metodológicas,

1 JULIÁ, S.: Historia social / sociología histórica. Madrid, Siglo XXI, 1989, pp. 10-40.

2 Decía E. P. Thompson, autor que ocupará un lugar protagonista en las páginas siguientes, que antes de la I Guerra Mundial imperó en las ciencias sociales el evolucionismo, mientras que después de aquel momento el voluntarismo se hizo con una posición hegemónica y propia de la Guerra Fría, por último, fue el estructuralismo (Thompson, E. P.: Miseria de la teoría. Barcelona, Crítica, 1981, p. 120 y ss). Respecto a la pregunta de con cuál período el historiador británico se sintió más identificado, baste decir que a él se atribuye la acuñación de la expresión historia desde abajo ya a la altura de 1966 (THOMPson, E. P.: «History from bellow», The Time Literary Suplement, (7 de abril de 1966), pp. 279-280). 
gran parte de las cuales se plantearon en el ámbito académico de los años 60 y 70 del siglo XX. Por lo tanto, muy atrás quedan las discusiones en torno a las que girará el texto, mas su recuperación se debe a que las dos disciplinas que aquí nos incumben, sociología e historia, hacen con frecuencia oídos sordos a los precedentes teóricos que las han conducido a preguntarse por las cuestiones actualmente en boga. Además, muchas de las cosas que aquí diremos parecerán materia poco novedosa a los expertos de los correspondientes campos, aun cuando no resulta tan corriente como debiera el que los unos estén familiarizados con lo que no son más que generalidades para los otros.

Vamos a optar aquí, grosso modo, por entender al individuo, desde el punto de vista metodológico siempre, como actor protagonista del proceso histórico-social, como el cabo del hilo por el que hay que empezar a tirar para arrastrar tras de sí el resto de constantes, constricciones, libertades, etcétera que configuran las comunidades humanas. Desde este planteamiento, adquiere sentido el que en distintos trabajos científicos se recuperen los testimonios de hombres y mujeres que vivieron y murieron hace muchos años, se entreviste a inmigrantes para conocer los avatares de sus biografías y sus formas de pensar, se trate de dar sentido a detalles en apariencia nimios que pertenecen al bagaje personal de la gente común. Pero imprescindible resulta también una cobertura teórica y por ello nos proponemos reunir, sólo de manera sucinta, un conjunto de aportaciones teóricas que puedan ser de ayuda para el encuadramiento de investigaciones empíricas en lo tocante al diálogo que se entabla entre el sujeto y la estructura.

Así, seguiremos un esquema expositivo en el que primeramente definiremos la palabra "acción» y sus implicaciones para la sociología; en segundo lugar, nos haremos eco de una polémica existente en torno al método científico puesto en práctica por el historiador, deteniéndonos en su capacidad, o impotencia, para alcanzar a conocer el sujeto; tarea del tercer apartado será conectar estas reflexiones previas con los conceptos de estructura y grupo para pasar, en último lugar, a la presentación de ciertas propuestas hechas por diversos estudiosos en torno a las nociones de red y estrategia.

Como podrá fácilmente comprobarse, apostamos en este artículo por aquel «tráfico de doble dirección» que proponía Edward H. Carr, al referirse a la beneficiosa práctica de conectar historia y sociología ${ }^{3}$, mal que las peculiaridades de la una y la otra pongan trabas a este método de trabajo.

\section{LA ACCIÓN SITUADA EN EL ORIGEN DE LAS SOCIEDADES}

Si el individuo, tanto en la actualidad como en el pasado, constituye el punto de arranque del análisis científico —así se estableció en la introducción-, sus ac-

${ }^{3}$ Véase CARR, E. H.: ¿Qué es la historia?: edición definitiva. Barcelona, Ariel, 2001. 
ciones han de pasar a ser objeto de observación detallada, ya que a partir de ellas se inferirá el funcionamiento del conjunto social. Una advertencia se hace pertinente para no dar pie a equívocos: la afirmación anterior debe entenderse en un contexto metodológico, nada tiene que ver con planteamientos de corte político, de hecho, el concepto de grupo y el de solidaridad hallarán más adelante un espacio propio en esta exposición.

Hecho este matiz, dentro del ámbito de los sociólogos, que será el que nos ocupe mayoritariamente en este apartado, la alternativa más adecuada para incorporar la noción de acción social a los trabajos de investigación consiste en adoptar un enfoque micro en el cual hallen cabida las «relaciones cara a cara». Asimismo entre los historiadores el desplazamiento de la idea de trayectoria colectiva hacia la de estrategias individuales ha supuesto una condición previa que ha facilitado la viabilidad de la sociología histórica ${ }^{4}$. El tomar partido por esta perspectiva de análisis permite los siguiente logros: 1) un buen estudio del individuo asegura una correcta interpretación de sus relaciones con los demás; 2) supone asimismo la vía más segura para que el recurso a la psicología sea fructífero y pueda evitarse así el error en que caen los estructuralistas, según los cuales las reacciones psicológicas colectivas son homogéneas; 3) permite, aunque es algo en lo que queda mucho por hacer, dibujar más fielmente los valores por los que se rige una sociedad ${ }^{5}$.

Ahora bien, lo que acabamos de hacer es delimitar el punto de observación correcto en el que debe fijarse el científico social, cosa muy distinta es que, a efectos expositivos, cuando trate de dar a conocer sus conclusiones, opte por elaborar modelos, los cuales no son más que construcciones intelectuales que simplifican la realidad a fin de destacar lo recurrente, lo constante y lo típico, que dichos modelos presentan en conjuntos de rasgos o atributos ${ }^{6}$. Prosigamos, así, con el desarrollo de la cuestión central de este apartado.

Para definir con mayor exactitud la idea de acción social, se hace preciso constatar ante todo que ésta es a un tiempo psíquica y social ${ }^{7}$. Los psicólogos sociales, ya desde la época de la Gestalt, han demostrado que las relaciones entre personas desconocidas, como principio de cualquier formación social, son regidas por un proceso en el que la representación del otro no resulta de una mera acumulación de observaciones directas sino que sufre un proceso de estructuración, en otras palabras, la interacción de los individuos pasa por la elaboración de unas imágenes, las cuales no dependen tan sólo de la persona, sino que la cultura a la que se pertenece y el medio ambiente de origen prestan buena parte de los ele-

4 BADIE, B.: «Análisis comparado y sociología histórica» Revista internacional de ciencias sociales, 133, (septiembre 1992), pp. 341-352 (la referencia en p. 347).

5 HECHTER, M.: «La teoría de la opción racional y la sociología histórica» Revista internacional de ciencias sociales, 133, (septiembre 1992), pp. 391-402.

6 BuRKe, P.: Sociología e historia. Madrid, Alianza, 1980, p. 41.

7 ROCHER, G.: Introducción a la sociología general. Barcelona, Herder, 1996, p. 21. 
mentos que componen las primeras impresiones y la imagen que uno se forja del otro $^{8}$.

Para el momento posterior a este «nacimiento de una sociedad», podríamos decir, gracias a la puesta en contacto de sus futuros miembros, los sociólogos han elaborado una compleja teoría de la acción. El segundo paso consistiría en desarrollar, a partir de la base de ese conocimiento mutuo estructurado, expectativas recíprocas que permitan al individuo conducirse en su comunidad de pertenencia, es decir, interiorizada ya la imagen del otro, se actuará de acuerdo con ella y se convertirá, por tanto, en el punto de referencia que guíe la toma de decisiones (cuerpo normativo) ${ }^{9}$. No obstante, lejos de ser éste un proceso estático, las relaciones sociales exigen una continua readaptación de los individuos, toda vez que, además, la interacción implica una influencia recíproca y una constante reformulación de los roles. Con el objeto de completar la definición de acción y de introducir cuestiones que nos ocuparán después, conviene recordar que, haciendo un uso riguroso de la terminología, la acción se refiere a las consecuencias que capta el actor, y el acto a aquellas que sólo se muestran visibles a un observador externo ${ }^{10}$.

Si bien parece haber acuerdo en estos principios generales, dos han sido las líneas básicas de interpretación dentro de la teoría sociológica clásica. Desde la óptica de Weber, para que la acción humana sea auténticamente social debe cumplir tres premisas: tener en cuenta el comportamiento de los demás (ya aludido), estar dotada de una significación o símbolo por el que la persona indique que ha comprendido las expectativas de los otros y, por último, estar influida por esas expectativas que el resto ha depositado en el sujeto ${ }^{11}$. Por su lado, Durkheim prefiere recurrir a un desdoblamiento según el cual la conciencia colectiva está constituida por el conjunto de maneras de obrar, de pensar y de sentir que integran la herencia de una sociedad, por tanto, es totalmente ajena a la persona y coacciona a la conciencia individual, esto es, la acción humana se verá siempre determinada, en mayor o menor grado, por un legado común que la coarta ${ }^{12}$. No ha de olvidarse

8 Ibidem, p. 17. Una explicación más detenida de este proceso, pero desde la perspectiva de los estereotipos sociales y centrada en la historia de la pobreza, puede encontrarse en GARCÍA SÁNCHEZ, M. A.: «La pobreza como construcción social en el Antiguo Régimen» Torre de los Lujanes, 51, (2003), pp. 97-124.

9 Una interesante corriente se ha afanado en los últimos años por vincular el nacimiento de las normas con los mecanismos de la acción colectiva en el seno de las redes sociales, tema de debate en las siguientes páginas, constituyendo tal propuesta una renovada visión de la movilización de grupos. A pesar de que queda fuera de nuestro objeto de interés, véase al respecto LINARES MARTíNEZ, F.: «Elección racional y normas sociales. Una vuelta de tuerca más» Revista Internacional de Sociología (RIS), 3. a época, 32, (mayo-agosto 2002), pp. 77-99.

10 LAMO DE ESPINOSA, E.: «El objeto de la sociología. Hecho social y consecuencias no intencionadas de la acción» REIS. Revista Española de Investigaciones Sociológicas, 48-49, (octubre-diciembre 1989), pp. 7-51, p. 24.

11 ROCHER, G.: Introducción..., pp. 22-25.

12 De entre la amplia bibliografía de estos dos autores, seleccionamos WEBER, M.: Economía y sociedad. Esbozo de sociología comprensiva. México, Fondo de Cultura Económica, 1964; del mismo autor, La ética protestante y el espíritu del capitalismo. Madrid, Losada, 2004; DURKHEIM, E.: El suicidio. Madrid, Alianza, 2001; y también, Las reglas del método sociológico. Buenos Aires, Schapire, 1965. 
que el «cosismo social» que suele achacarse a Durkheim es muy discutido aún hoy al dotar de vida propia a ciertos recursos explicativos que carecen de tal autonomía, en una maniobra que desvirtúa su sentido originario.

Las opciones interpretativas aquí presentadas a vuela pluma, por último, se conjugan en una suerte de término medio, bastante exitoso, conocido como orientación normativa de la acción social. Este ingenio reconoce la fuerza coercitiva de las estructuras, pero sin convertirlas en agentes determinantes, al mismo tiempo que permite una iniciativa propia a los individuos. Bajo ningún concepto puede obviarse, pues, la existencia de normas colectivas, las cuales emanan de esa interacción con la que abríamos el artículo y que requiere unas reglas del juego capaces de asegurar la previsión de las actuaciones ajenas y la reciprocidad ${ }^{13}$.

Los siguientes escalones que habríamos de subir para completar esta teoría pasarían por abordar conceptos tales como el de rol, estatus, proceso de socialización, sistema, valores, etc. En fin, nos interesaba para nuestro objeto de estudio únicamente aproximarnos al origen sociológico de la idea de acción. Aunque de este modo hemos definido de refilón al sujeto, pues es el motor de cualquier acción, sobre este punto, no obstante, habrá que profundizar a continuación, ya que de lo contrario correremos el riesgo de no entender de forma adecuada las propuestas recientes que distintos profesionales de las ciencias humanas han lanzado.

\section{EL MÉTODO HISTÓRICO Y EL CONOCIMIENTO DEL SUJETO}

Se han hecho ya algunas referencias al objeto metodológico y a las precauciones que han de tomarse cuando se trata de distinguirlo del producto final que se escribe tras una investigación. Corresponde ahora detenernos algo más en esta dicotomía pero centrándonos de lleno en el campo de la historia. Para ello, las reflexiones de un filósofo, Althusser, y un historiador, Thompson, nos serán de gran ayuda.

A grandes rasgos podemos decir que, según el primero, el empirismo no resulta un método adecuado para afrontar el conocimiento del pasado y además, en consonancia con esta afirmación, el individuo, dentro de las sociedades que nos

${ }_{13}$ Quedan en el tintero multitud de enfoques que ayudarían a comprender este problema. En primer lugar, la vasta obra de Parsons ilustra en lo concerniente al funcionamiento del sistema y a la participación de los individuos en el mismo. Véase PARSONS, T.: El sistema social. Madrid, Alianza, 1999; así como, Action Theory and the Human Condition. Nueva York, The Free Press, 1978. Por otro lado, el interaccionismo simbólico fue una corriente inaugurada por Mead y que ha cosechado notable número de seguidores a partir de postulados psicológicos y lingüísticos. Véase MEAD, G. H.: Espíritu, persona y sociedad desde el punto de vista del conductismo social. Barcelona, Paidós, 1982 y GoffmAN, E.: La presentación de la persona en la vida cotidiana. Madrid, Martínez de Murguía, 1987. Por último, la racionalidad de la acción fue objeto de reflexión por parte de J. ELSTER, como prueba su trabajo Lógica y sociedad: contradicciones y mundos posibles. Barcelona, Gedisa, 1994. 
han precedido, no se constituyó como un sujeto activo, sino que el transcurrir de la historia ha dependido en todo momento de unos vectores que lo han sobrepasado. Epistemológicamente, añade, la historia real es incognoscible, de tal suerte que no ha lugar un método científico-empirista ${ }^{14}$. A todo ello repuso Thompson, por su parte, que Althusser no es capaz de distinguir entre el empirismo y el diálogo empíri$\mathrm{co}$, y que en su concepción estructuralista no tiene cabida el dinamismo del cambio histórico ${ }^{15}$. Uniéndose al debate, Anderson criticó a las obras de Thompson su excesivo idealismo y la insolente laxitud con la que hace uso de los conceptos marxistas, reservando para Althusser un reproche, para nada baladí, consistente en no saber entender que en historia el objeto y el sujeto de conocimiento coinciden. Desarrollemos con más calma este denso esquema que acabamos de exponer.

Por lo pronto, intentaremos, siempre que sea posible, dejar de lado la cuestión de la cientificidad de la historia, algo superado hoy pero que subyace en todos estos juicios, para centrarnos mejor en los problemas de método que se detectan en el cruce de acusaciones arriba bosquejado. Así, mientras que para Thompson las fuentes históricas deben pasar por el filtro del análisis del historiador para obtener un dato fiable, Althusser desconfía de tal correlación, en tanto en cuanto la experimentación y la verificación, simplemente, no son factibles al tratar las sociedades del pasado. Aunque el británico insiste en que la historia se ha dotado de unas herramientas capaces de interrogar adecuadamente los testimonios documentales que nos han sido legados, para el filósofo francés, por el contrario, eso no es más que una muestra de «ideología empírica».

Tal desacuerdo atañe directamente al fin que persigue el presente trabajo pues, si no se puede conocer el sujeto de la historia a través de las fuentes, otras han de ser las preocupaciones del historiador, consistentes antes en la indagación de los vectores estructurales que han determinado el porvenir de unos individuos pasivos o, en otras palabras, meros objetos. Por tanto, la fase metodológica de la observación resulta de capital importancia puesto que, según sea entendida, para unos parecerá pertinente la investigación particular de personas con nombres propios (si bien no por ello pertenecientes a las élites), mientras que para otros se estaría incurriendo entonces en un error de partida.

\footnotetext{
${ }^{14}$ Althusser, L. y Balibar, E.: Para leer El Capital. México, Siglo XXI, 1969; de los mismos, Écrits philosophiques et politiques. París, IMEC, 1994. Quizá más amable se nos muestre Althusser, quien será objeto de crítica a lo largo de estas páginas, si tenemos en cuenta cuál era su intención al elaborar los recién apuntados principios: «En el curso de su elaboración teórica y en un esfuerzo por superar el determinismo económico, Althusser presenta una versión revisada del modelo base-superestructura (...). En él, el modo de producción se entiende como constituido por tres niveles relativamente autónomos (económico, político e ideológico) en el que el nivel económico sólo es determinante en última instancia» (KAYE, H.: Los historiadores marxistas británicos. Un análisis introductorio. Zaragoza, Prensas Universitas, 1989, p. 189).

${ }_{15}$ Thompson, E. P.: Miseria .... Véase también del mismo autor, The making of the English working class. Londres, Penguin, 1991 y William Morris, romantic to revolutionary. Londres, Lawrence \& Wishart, 1955.
} 
Profundizando algo más en estas ideas, la enjundiosa frase de Althusser, el conocimiento de la historia no es histórico en mayor grado que pueda ser dulce el conocimiento del azúcar, prueba una vez más el no reconocimiento por su parte de un método propiamente histórico, de tal suerte que en su universo el fin (la Historia, lo que realmente ocurrió) se confunde con el medio (el conocimiento histórico, el oficio de historiador). El rebatir esta aseveración ocupa buena parte de las páginas de Miseria de la teoría pero, resumiendo en pocas líneas, el argumento de Thompson viene a defender que, aunque sí es cierto que el historiador es un sujeto que analiza a otro sujeto ubicado en el pasado, no resulta admisible el que la historia «real» y el conocimiento histórico sean lo mismo ${ }^{16}$. La defensa acérrima que lleva a cabo este autor de un método particular para la historia, como fruto de años de investigación en archivos, se rebela contra los que piensan que «si la explicación histórica no puede ser el Todo, entonces no es Nada »17.

En este sentido, resultaría desacertado escamotear la alusión a la doctrina marxista en la que se inspiró con profusión la práctica totalidad de los componentes de la escuela a la que pertenece Thompson. No obstante, continuando con nuestra línea argumental, el autor de La formación de la clase obrera insistió en que el método del materialismo histórico es distinto a la doctrina promovida por Marx, incluso, esta última puede terminar engendrando interpretaciones históricas desatinadas en las que no halle cabida el agente humano: «si dejamos que la filosofía trate de abstraer los conceptos respecto de las prácticas y construya a partir de ellos un Hogar para la Teoría independientemente de éstas, y además lejos de todo diálogo con el objeto de la teoría, entonces tendremos... jel teatro de Althusser! » ${ }^{18}$. Así, Thompson mantuvo una lucha intelectual contra esas variedades de marxismo y de ciencia social que están caracterizadas por el determinismo económico y la negación de la acción humana. Es más, insistió en que el marxismo puede entenderse mejor como una teoría de la historia, no como leyes de la historia, en las que los seres humanos viven patrones de desarrollo predeterminados, y en que la clase es un concepto histórico, no sólo una categoría o construcción sociológica ${ }^{19}$.

Quizás el traer a colación el ejemplo de las relaciones de producción sea el modo más gráfico de entender cómo un enfoque netamente estructuralista, así como explicaciones doctrinarias, entorpecen el conocimiento del sujeto y la ac-

16 Thompson, E. P.: Miseria..., p. 38.

17 «Por lógica histórica entiendo un método lógico de investigación adecuado a los materiales históricos, concebido, en el mayor grado posible, para contrastar hipótesis relativas a estructuras, causaciones, etc., y para eliminar procedimientos autoconfirmatorios (ejemplos, ilustraciones) [...] El conocimiento histórico es, por su naturaleza, a) provisional e incompleto, aunque no por ello falso, b) selectivo, aunque no por ello falso, c) limitado y definido por las preguntas formuladas a los datos empíricos (y los conceptos que informan estas preguntas) y, por lo tanto, sólo verdadero dentro del campo así definido» (Thompson, E. P.: Miseria..., pp. 67-68). A nuestro modo de ver, esta cita sintetiza de manera inmejorable las claves del tan discutido método histórico.

18 Thompson, E. P.: Miseria..., p. 76.

19 KAYE, H.: Los historiadores..., p. 160. 
ción: «La estructura de las relaciones de producción determina unos puestos y unas funciones - sostiene Althusser - que son ocupados y asumidos por agentes de la producción que nunca son más que los ocupantes de esos puestos, en la medida que son los portadores (Träger) de esas funciones. Los verdaderos sujetos (en el sentido de sujetos constituyentes de los procesos) no son pues estos ocupantes o estos funcionarios; no son pues, contrariamente a todas las apariencias, las evidencias de lo dado de la antropología ingenua, los individuos concretos, los hombres reales; sino que son la definición y la distribución de estos puestos y de estas funciones. Los verdaderos sujetos son pues estos definidores $y$ estos distribuidores: las relaciones de producción (y las relaciones sociales políticas o ideológicas). Pero, dado que se trata de relaciones, no pueden pensarse bajo la categoría de sujeto ${ }^{20}$. Pocos manifiestos hallaremos tan ilustrativos como este del estructuralismo más radical pero, a la vez también, consecuente consigo mismo y meditado.

En respuesta, el propio Thompson matiza, una vez más, que las personas se someten, claro está, a ciertos condicionantes pertenecientes a la estructura social, pero en ningún caso llegan éstos a ser tan potentes como para anular su capacidad de acción ${ }^{21}$. Ahora bien, no puede olvidarse que sus propuestas han estado en el punto de mira de ciertas críticas debidamente fundamentadas. En su huida del estructuralismo más reduccionista, afirman algunos autores, llegó a sobredimensionar el papel desempeñado por el cambio social en la historia, como si cualquier ralentización del ritmo evolutivo de las sociedades hiciera tambalear su visión epistemológica. Hay quienes, tal es el caso de Meiksins Wood, detectan en la obra de Thompson la falta de un espacio en el que contemplar impulsos regresivos dentro de la conciencia popular, estancamientos en el devenir histórico, ausencias de conflictividad, en fin, momentos de estabilidad. Por tanto, resulta contraproducente desechar cualquier evidencia que no apunte hacia la acción de los individuos, toda vez que tal actitud del historiador británico responde a un intento de conceder un lugar propio a las clases sociales inferiores, tradicionalmente ignoradas. Se ha llegado a sostener desde diversas corrientes académicas que, a pesar del humanismo que Thompson intenta imprimir en sus obras, incurre sin embargo con frecuencia en argumentaciones deterministas. A este respecto, Sewell opina que la estructura desempeña un papel insustituible en la epistemología thompsoniana, eso sí, no como ley sino como lógica que explica el modo en que «la causalidad va

20 Thompson, E. P.: Miseria..., p. 226. Las cursivas se toman de Lire le capital, II (versión española: Althusser, L. y Balibar, E.: Para leer...) Dos adecuadas síntesis, desde el enfoque de la teoría sociológica, que ilustran sobre las distintas propuestas existentes en torno a la desigualdad social (Davis, Moore, Dahrendorf y Wright entre otros) son las de CROMPTON, R.: Clase y estratificación. Una introducción a los debates actuales. Madrid, Tecnos, 1993 y FEITo Alonso, R.: Estructura social contemporánea. Las clases sociales en los países industrializados. Madrid, Siglo XXI, 1995.

${ }^{21}$ En este caso será N. Poulantzas el que nos ofrezca un término medio según el cual, continuando con el ejemplo seleccionado, el modo de producción debe entenderse como un «objeto abstracto formal» que sólo existe en la realidad cuando pasa a ser contenido por las formaciones sociales (MEIKSINS Wood, E.: «Entre las fisuras teóricas: E. P. Thompson y el debate sobre la base y la superestructura» Historia Social, 18, (invierno 1994), pp. 103-124, p. 108). 
de las relaciones económicas a la conciencia pasando por la experiencia social»22.

Cerramos de este modo una discusión que hubiera dado para muchas más páginas puesto que se ha erigido, a nuestro juicio, en una de las polémicas historiográficas más fructíferas de las últimas décadas, de tal suerte que podrían haberse abordado aquí preguntas tales como, por ejemplo, la derivación del marxismo británico hacia la historia cultural (o «moralismo romanticista» a los ojos menos benevolentes de Anderson), sus reflexiones en torno al concepto de clase ${ }^{23}$, así como el trasfondo personal que subyace bajo los desacuerdos académicos ${ }^{24}$. Por todo ello, creemos haber podido demostrar la vigencia de unas obras, en ningún caso trasnochadas, a pesar de lo que pueda parecer a primera vista, y también la fertilidad de sus apreciaciones acerca del oficio de historiador según hoy se entiende.

\section{ESTRUCTURA, GRUPO, INDIVIDUO: ¿TRES ELEMENTOS IRRECONCILIABLES?}

Toca el turno ahora de abordar el valor de la estructura desde una perspectiva distinta, no tanto ya como cuestión metodológica, sino más bien epistemológica. Para ello, dos corrientes, la individualista y la situacionista, servirán de guías a partir de las cuales avanzaremos hacia el concepto de grupo como punto nodal entre la estructura y el individuo.

Pues bien, una definición bastante aséptica del concepto de estructura podría ser la siguiente: «modo en que las partes de un sistema social (individuos, organizaciones, grupos) se relacionan entre sí y forman el todo, pudiendo eventualmente presentar sucesivas y diferentes conformaciones o transformaciones, sin que sea por ello otro el sistema social en cuestión ${ }^{25}$. De acuerdo con este principio, cualquier modificación de los elementos que componen la estructura repercutirá en el resto, además, toda observación sociológica debe hallar cabida en el modelo estructural para que éste sea coherente $y$, por último, al tratarse de un

22 SeWeLL, W. H., Jr.: «Cómo se forman las clases: reflexiones críticas en torno a la teoría de E. P. Thompson sobre la formación de la clase obrera» Historia Social, 18, (invierno 1994), pp. 77-102, p. 83. Del mismo, véase también, «A theory of structure: Duality, agency, and transformation» American Journal of Sociology, 98:1, (1993), pp. 1-29.

23 «La propuesta de que las clases se constituyen por modos de producción puede con facilidad conducir a la práctica de identificar una clase como una estructura objetiva en sí misma» (KAYE, H.: Los historiadores..., p. 218).

24 El cruce de opiniones tiene por escenario la publicación anual de corte marxista que editaban R. Miliband y J. Saville, Socialist Register, y la revista New Left Review, concretamente, Thompson critica los postulados de Anderson en el Socialist Register de 1965 y a L. Kolakowski en el mismo lugar pero en 1973; por su parte, Anderson replicará en el número 33 de New Left Review (1966) y Kolakowski en Socialist Register (1974).

25 Beltrán Villalva, M.: «Sobre la noción de estructura social» Revista Internacional de Sociología, 3. ${ }^{a}$ época, 30, (septiembre-diciembre 2001), pp. 7-28, p. 8. Un trabajo clásico al respecto es el de PomiAN, K.: «La historia de las estructuras», en Le Goff, J., CHARTIER, R. y ReVEL, J. (dirs.): Diccionario del saber moderno. La nueva historia. Bilbao, Mensajero, 1988, pp. 196-221. 
constructo interrelacionado, sería posible predecir qué consecuencias tendría la variación aislada de una de sus partes. Tal sería la fórmula defendida por aquellos que depositan su mayor confianza en la utilidad del método estructuralista. No obstante, y gracias a la proyección del mismo hacia la práctica de la investigación, el cambio dentro de la estructura ha sido ampliamente reconocido en los últimos años, a la vez que se ha ido relegando a un segundo plano los mecanismos sociales de equilibrio y autoperpetuación ${ }^{26}$.

Es más, visiones recientes de la estructura, y comenzamos a entrelazar perspectivas, han tendido a incorporar la idea de acción social concediendo un papel protagonista al individuo como sujeto participante en el andamiaje que sostiene cualquier comunidad humana, de modo que la estructura debe ser una inferencia hecha desde la realidad empíricamente observable, a fin de sortear así los reparos que suscitaban las rotundas propuestas de Althusser. Para Beltrán Villalva, por ejemplo, el concepto que estamos procurando definir se explica mediante las relaciones establecidas entre las partes o individuos que constituyen el todo y propone este autor que el sistema social se organiza gracias a una serie de subsistemas articulados entre sí (demografía, cultura, economía, política e historia) dentro de los cuales cada «elemento, parte o individuo» tiene una posición determinada ${ }^{27}$. De tal forma, el individuo se ve imbricado en una nueva propuesta analítica donde adquiere un papel protagonista.

Beltrán Villalva, no obstante, nos previene de que el individualismo metodológico que acabamos de proponer, aun atractivo y renovador en el ámbito de las ciencias sociales, puede hacernos caer en la trampa - no es la primera vez que la mencionamos- de creer que la sociedad es una mera suma de personas. Ahora bien, desde distintas disciplinas se ha interiorizado debidamente esta precaución y sirvan de ejemplo los trabajos de Buchanan ${ }^{28}$ e Infantino ${ }^{29}$ en el campo de la economía, Homans ${ }^{30}$ en el de la psicología social o Coleman ${ }^{31}$, autor que, a modo de ejemplo de estas posturas académicas, defiende «que la interacción entre individuos produce fenómenos emergentes a nivel del sistema, esto es, fenómenos no deseados ni previstos por los individuos. Más aún, una explicación concreta no tiene por qué descender hasta el nivel individual para ser satisfactoria ${ }^{32}$. Por su parte, Hayek ${ }^{33}$ opina que el orden social no es producto de la Razón sino que deriva de las acciones individuales, por tanto, no es intencional ni consciente.

26 LUHMANN, N.: Soziologische Aufklärung. Opladen, 1974.

27 Beltrán Villalva, M.: «Sobre la noción ...», pp. 13-14.

28 BuChanan, J. M.: Economía y política. Escritos seleccionados. Valencia, Universitat de Valencia, 1988.

29 INFANTINO, I.: L'ordine senza piano. Le ragioni dell'individualismo metodologico. Roma, La Nuova Italia Scientifica, 1995.

30 Homans, G. H.: «Social behaviour as exchange» The American Journal of Sociology, LXII, (mayo 1958), pp. 597-606.

31 Coleman, J. S.: Foundations of social theory. Cambridge, Harvard University Press, 1990.

32 Citado en Beltrán Villalva, M.: «Sobre la noción ...», p. 19.

33 HAYEK, F. A.: Derecho, legislación y libertad: una nueva formulación de los principios liberales de la justicia y de la política economía. Madrid, Unión Editorial, 1988. 
Todos ellos participan, pues, de un individualismo matizado que cuenta con una alternativa más, a nuestro juicio, no del todo opuesta, como es la elaborada por A. Giddens. Parte este sociólogo de la base de que la persona, como agente motor de los acontecimientos, cuenta con un bagaje de conceptos (llega a emplear la peligrosa expresión «portador de conceptos»" demasiado cercana a la de Träger) que le permite reflexionar sobre su propia conducta (lo cual define la experiencia humana y diferencia a ésta de la posible acción animal). En este sentido y enlazando con el epígrafe anterior, considera que la trascendencia del concepto de acción defendido por Thompson pasa por los siguientes puntos ${ }^{35}$ :

- Gracias a él, el estudio histórico no ignora a los que carecen de privilegios.

- Tampoco pasa por alto los acontecimientos cotidianos.

- Promueve la contingencia de la historia, es decir, la carencia de una teleología.

- «Los seres humanos hacen su propia historia de un modo activo tanto como son hechos por ella» ${ }^{36}$.

Sin embargo, con estas reflexiones como prólogo de su trabajo, la aportación propia de Giddens consiste en el concepto de dualidad de la estructura ${ }^{37}$. Cree que no es cierto que el actor, en las coordenadas thompsonianas, sea siempre capaz de «actuar de otra manera» (volvemos a la discusión determinismo-libre albedrío) ni, por contra, que las instituciones sociales logren monopolizar la vida del individuo. Así, acción y estructura se compenetran descansando sobre las siguientes bases: 1) cualquier acción humana se desarrolla en el contexto de un sólido entramado institucional; 2) las instituciones están enraizadas en la vida cotidiana; 3) la sociedad se dota de mecanismos que penalizan y anulan parte de las opciones a las cuales, teóricamente, el actor tendría acceso; 4) por sí mismos los grupos sociales se imponen unos sobre otros y reducen el campo de acción del individuo; 5) las consecuencias de la acción humana no siempre son previsibles cuando ésta se idea. Sewell acierta en el juicio que le sugiere la propuesta giddesiana: «[...] descosifica la estructura y la convierte en algo no menos humano que la acción; en lugar de ser antagonistas, para él van indisolublemente unidas: la acción y la estructura presuponen la una a la otra. Según él, las estructuras son, al mismo

${ }^{34}$ GIDDENS, A.: «Fuera del mecanicismo: E. P. Thompson sobre conciencia e historia» Historia Social, 18, (invierno 1994), pp. 153-170, p. 163.

35 «El defecto básico de gran parte de los escritos sobre acción y estructura, incluyendo los de Thompson y Anderson, es suponer que o bien el individuo tiene primacía sobre la sociedad (modos de producción/formación social) o a la inversa. Thompson se decanta en favor de la realidad de los individuos, o por lo menos de la experiencia individual [...] Anderson se encuentra más cerca del canon sociológico ortodoxo: las sociedades, o las formaciones sociales, son anteriores a la existencia de los individuos y determinan sus actitudes y modos de conducta característicos» (GIDDENS, A.: «Fuera del mecanicismo ...", p. 166).

36 GidDENS, A.: Ibidem, p. 164.

${ }^{37}$ GIDDENS, A.: Central problems in social theory: Action, structure and contradiction in social analysis. Londres, Macmillan, 1979; y Constitution of society: Outline of the theory of structuration. Cambridge, Polity Press, 1984. 
tiempo, el médium y el resultado de las interacciones humanas. Son transformadas pero también reproducidas por los agentes. No sólo determinan o limitan, sino que además posibilitan: los agentes no podrían existir sin las estructuras que establecen sus limitaciones y posibilidades y las estructuras no podrían existir sin los agentes que las materializan y/o las transforman ${ }^{38}$.

Al respecto, Beltrán Villalva llama la atención sobre cómo uno de los resultados no intencionales de la conducta de los individuos consiste en reproducir la estructura social, aparte de que, simultáneamente, la estructura esté presente en toda acción promovida por las personas. Este doble juego es lo que Giddens denomina dualidad de la estructura: «La estructura que ha de ser reproducida está presente en la acción social, y es reproducida por ésta. No hay aquí, pues, mano invisible alguna, ni autorregulación social» ${ }^{39}$. Por lo tanto, ajeno al universo de Althusser en el que la estructura era restrictiva, Giddens opta por una perspectiva en la que la acción se ve capacitada gracias al antaño opresor andamiaje social.

Igualmente conciliador se presenta, por otro lado, Bourdieu, el cual no duda en afirmar que en la vida cotidiana disponemos de un acervo de recursos para la acción adquirido en el proceso de socialización. Tal bagaje proporciona una serie de salidas en cada situación que van constituyendo hábitos sociales ${ }^{40}$. Cada clase social cuenta con hábitos propios forjados a través del tiempo, por tanto, si bien la estructura no determina la actuación del individuo, sí que la constriñe en cierto modo, puesto que empuja a los individuos con similares hábitos a conformarse en un grupo homogéneo.

Como ha podido comprobarse, con Giddens y Bourdieu, hemos evolucionado desde concepciones individualistas de la acción a concepciones situacionistas más preocupadas por insertar al individuo en un contexto concreto. Antes de pasar a la noción de grupo, recogemos a continuación una cita que refiere una opción teórica mesurada y en buena medida satisfactoria, a la altura de la autoridad académica que la escribe.

«Las necesidades prácticas de la vida social [...] obligan a los hombres a vivir a través de coaliciones, instituciones, clanes y comunidades que adquieren una autonomía específica. Esta autonomía relativa les viene conferida por el constante proceso de reificación que sufre la interacción humana [...], reificación que no es nunca permanente, que está siempre en doble proceso de estructuración y desestructuración, y ello en gran manera de un modo conflictivo. El estudio objetivo de las entidades reificadas, hijas de la interacción humana [...] es posible, más aún, necesario, y no ha sido otro el campo tradicional de la empresa sociológica ${ }^{41}$.

38 SEWELL, W. H., Jr.: «Cómo se forman ...», p. 92.

39 Beltrán Villalva, M.: «Sobre la noción ...», p. 22.

40 Beltrán Villalva, M.: Ibidem, p. 23.

41 GINER, S.: «Intenciones humanas y estructuras sociales: aproximación crítica a la lógica situacional» Cuadernos Económicos de ICE, 3-4, pp. 110-145, p. 131, citado en BELTRán VilLALVA, M.: «Sobre la noción ...», p. 25. 
Finalmente, el último punto que resta para cerrar este apartado tiene por fin salvar ese vacío que parece mediar entre el individuo y la estructura mediante el recurso al concepto de grupo, en tanto que vehículo que imbrica a los individuos entre sí y que facilita al investigador captar mejor las bases sobre las cuales se asienta la estructura. Cambiaremos el tono de nuestro hilo argumental y pasaremos de unas reflexiones de corte teórico a otras más apegadas a la práctica empírica. Es por ello que se muestra adecuada la recuperación de la teoría del campo social y de la dinámica de grupos como instrumentos de investigación. Fue Lewin, por cierto, el científico que ya en la década de los años 30 y 40 del siglo XX puso sobre el tapete la teoría del campo social, la cual puede sintetizarse grosso modo en lo siguiente: la conducta (acción) es función de la personalidad y del entorno, a su vez, la estructura del entorno depende de los deseos y de las necesidades de la persona ${ }^{42}$. «La persona no está, pues, fuera de la situación, sino que forma parte de la misma, y, en el seno de la situación, no hay frontera inmutable y netamente limitada entre las conciencias individuales y su entorno" ${ }^{43}$.

Ahora bien, el diálogo del individuo con la estructura, según el enfoque que nos ocupa, requiere de un importante nexo, cual es el grupo. La integración en el entorno se produce, en la práctica, a través de un entramado de relaciones humanas. «Toda dinámica de grupo es la resultante del conjunto de las interacciones dentro de un espacio psicológico-social [...] El grupo es para el individuo un instrumento. Es decir, que el individuo utiliza, más o menos conscientemente, el grupo y las relaciones sociales que él sostiene en su grupo, como instrumentos para satisfacer sus necesidades físicas o sus aspiraciones sociales» ${ }^{44}$. En efecto, esta búsqueda del interés propio granjeado por medio de las relaciones con los demás será una línea interpretativa constante tanto entre los sociólogos como entre los historiadores recientes, ya lo veremos en el siguiente epígrafe. No ha de entenderse, sin embargo, ese toma y daca realizado en el seno del colectivo de pertenencia desde un punto de vista meramente material sino que también la negociación y el intercambio se extienden a los significados sociales y a la ocupación de roles ${ }^{45}$. De este modo, se ha desplazado el centro de atención de estas páginas hacia el grupo. Comenzábamos el epígrafe preguntándonos por el significado de la palabra estructura

42 LeWIN, K.: Field theory in social science: Selected theoretical papers. Nueva York, Harper, 1951. Véase también COOLEY, Ch.: On self and social organization. Chicago, University of Chicago Press, 1998 y MAYO, E.: The social problems of an industrial civilization: With an appendix on the political problem. Londres, Routledge \& Kegan Paul, 1975.

43 RocheR, G.: Introducción..., p. 33. Aplicado al análisis micro de las agrupaciones humanas, estas definiciones pueden recordarnos a las opciones sostenidas por Giddens y Bourdieu que venimos de explicar y es que ciertamente K. Lewin vivió también una etapa crítica para el individualismo, pero esta vez la acaecida en los años 30, bajo el influjo de Freud. Propio del contexto del behaviourismo había sido estudiar la influencia del grupo sobre la persona, mas poco a poco también se había optado por invertir esfuerzos en conocer la forma en que la persona, a menudo bajo la forma de líder, puede influir en el grupo. Se abría así un abanico de posibilidades al científico que en buena medida ha llegado hasta nuestros días. Véase el capítulo 2 de MAILHIOT, B.: Dinámica y génesis de grupos. Madrid, Marova, 1980, titulado «Una etapa decisiva para la psicología social».

44 MAILHIOT, B.: Dinámica..., pp. 50-54.

45 Ayestarán, S. (dir.): El grupo como construcción social. Rubí, Plural, 1996, p. 191. 
y pasábamos después a las perspectivas individualista y situacionista. Hemos concluido con una referencia a la teoría del campo social, apenas unos trazos, que nos servirá de introducción para la última parte del artículo, en este caso, dedicada a las aportaciones relativas al funcionamiento interno de las redes.

\section{ALGUNAS PROPUESTAS DE ANÁLISIS}

Cuatro son las opciones metodológicas que presentamos a continuación: intercambio, redes, cohesión social y estrategias. Citadas a menudo, objeto de sustanciosas reflexiones, carecen sin embargo de sentido si no se tienen en cuenta los puntos de referencia que hemos expuesto en las anteriores secciones. Debe añadirse que, exceptuando el de estrategia, los demás conceptos no abundan en los escritos de los historiadores, aun cuando cuentan con una larga tradición entre los sociólogos.

Pues bien, desde un magnífico estudio etnográfico publicado en 1974 Boissevain afirmaba que «las configuraciones sociales tales como coaliciones, grupos, instituciones y sociedad deben ser vistas como redes de personas que llevan a cabo elecciones compitiendo por escasos y valiosos recursos ${ }^{46}$. Para los teóricos del intercambio social, las relaciones sociales implican el toma y daca de bienes valiosos, ya sean materiales o simbólicos, y dentro de su esquema metodológico, además, el actor es constantemente influido por la necesidad de satisfacer sus intereses, tarea esta en la que queda sujeto a un mecanismo de castigo y recompensa que regula todo intercambio ${ }^{47}$. Si bien tal podría ser el patrón básico de un modo peculiar de acercarse al problema, cada autor ha impreso sus variantes particulares, pudiendo citarse los casos de Homans, Blau y Emerson, entre muchos otros $^{48}$.

En segundo lugar, el análisis de redes, estrechamente relacionado con la perspectiva anterior, se ha basado en estudios más empíricos acerca de grupos concretos y de los vínculos trabados por sus miembros, toda vez que ha de reconocerse la existencia de una tendencia estructuralista, entre los cultivadores de esta corriente, ocupada en discernir cómo los modelos generados por las propias redes llegan a constreñir el comportamiento de los individuos que viven insertos en ellas $^{49}$.

${ }^{46}$ BoIssevain, J.: Friends of friends. Networks, manipulators and coalitions. Bristol, Oxford Basil Blackwell, 1974, p. 9 (traducción propia).

${ }_{47}$ COOK, K. S. Y WHITMEYER, J. M.: «Two approaches to social structure: Exchange theory and network analysis» Annual Review of Sociology, 18, (1992), pp. 109-127, p. 114.

48 Un repaso de sus trabajos puede encontrarse en COOK, K. S. Y WHITMEYER, J. M.: «Two approaches ...», pp. 111-114. En la misma línea de análisis debe situarse la ya referida dinámica de grupos.

49 REQUena SANTOS, F.: «El concepto de red social» REIS. Revista Española de Investigaciones Sociológicas, 48, (octubre-diciembre 1989), pp. 137-152. Véase también REQUENA SANTOS, F.: Análisis de redes sociales. Orígenes, teorías y aplicaciones. Madrid, Centro de Investigaciones Sociológicas, 2003. Por otra parte, una breve síntesis acerca del tema puede hallarse en la comunicación GARCíA SÁNCHEZ, M. A.: 
Cook y Whitmeyer, los autores que venimos siguiendo, apuestan por una síntesis de los dos enfoques presentados: nos referimos a la Network Exchange Theory. La clave para tener éxito en este ejercicio, afirman, reside en contar con un adecuado concepto de actor individual, por tanto, nuestro detenimiento en dicha cuestión no parece haber sido en vano. Así, la búsqueda del interés personal, propia de la teoría del intercambio, casa también con la perspectiva de las redes; por añadido, qué duda cabe, la transacción de favores, bienes materiales, significados simbólicos, etcétera se desarrolla invariablemente en el escenario de una comunidad cuyos miembros están vinculados entre sín ${ }^{50}$. La teoría del intercambio sostiene que las interacciones, y por extensión, los efectos de las redes sobre la acción y la estructura, ocurren únicamente debido al valor del intercambio de los artículos transferidos (que pueden ser materiales, simbólicos, de información, etcétera). Cuando las relaciones de intercambio se excluyen, los resultados tienen una alta probabilidad de ser falsos, si no un error ${ }^{51}$. Nos hallamos ante un enfoque que dirige la atención del científico social hacia una de las claves que nunca ha de descuidarse en una investigación.

Tomando estas corrientes como punto de partida, otra escuela de sociólogos se ha interesado, en tercer lugar, por intentar calibrar el grado de cohesión con el que están trabadas esas redes a las que hacemos alusión de manera constante. Fue Granovetter quien llevó a cabo trabajos pioneros en este campo, abriendo así una senda que ha dado notables frutos en los últimos años ${ }^{52}$. «Analíticamente, el concepto de solidaridad puede ser dividido entre un componente ideal, referido a la identificación de los miembros con una colectividad, y un componente relacional, referido a las conexiones observadas en los miembros de la colectividad ${ }^{53}$. Podemos definir la cohesión estructural como la medida del componente relacional. Asimismo social embeddedness constituye una expresión que, aparte de hallar difícil traducción al castellano («incrustación»), se refiere a la importancia de las redes sociales para la acción, esto es, los individuos pertenecientes a grupos con una fuerte cohesión disponen de un mayor número de recursos que el resto ${ }^{54}$.

\footnotetext{
"Análisis de redes sociales: una propuesta metodológica», Congreso Ocio y vida cotidiana en el mundo hispánico, siglos XVI-XVIII, Universidad de Sevilla-Dpto. de Historia Moderna, 2003 (en prensa).

50 Sin embargo, los teóricos que se han ocupado del análisis de redes tienden a ser más restrictivos con la noción de intercambio, mostrándose cautelosos a la hora de calificar como tal cualquier vínculo observable entre los componentes de un grupo.

${ }^{51}$ COOK, K. S. Y WHITMEYER, J. M.: «TWO ...», pp. 120-121.

52 GranovetTer, M. S.: «The strength of weak ties» American Journal of Sociology, 78;6, (1973), pp. 1.360-1.380; del mismo autor, «The strength of weak ties. A network theory revised», en MARSDEN, P. V. y LIN, N. (eds.): Social structure and network analysis. Beverly Hills, SAGE, 1982, pp. 105-130 (versión traducida en REQUENA SANTOS, F.: Análisis...)

53 MoOdY, J. y WHITE, D. R.: «Structural cohesion and embeddedness: A hierarchical concept of social groups" American Sociological Review, 68, (febrero 2003), pp. 103-127, p. 104 (traducción propia).

54 GranovetTer, M. S.: «Economic action and social structure: The problem of embeddedness» American Journal of Sociology, 91:3, (1985), pp. 481-510 (versión traducida en REQUENA SANTOS, F.: Análisis...)
} 
Contamos, así pues, con una serie de herramientas que pueden aplicarse a todo tipo de grupos, no sólo a los de pequeñas dimensiones, como se venía haciendo hasta ahora. Se trata de una completa propuesta metodológica que, de ponerse en práctica exitosamente, dinamizaría de forma notable el campo de la historia, ahora bien, su complejidad y farragosas fórmulas matemáticas disuaden con frecuencia de tal propósito. Aun así, nociones como las de path, node, nest,... empleadas para describir la posición y papel de cada actor dentro de la red bien merecen una atención por parte de todos los profesionales involucrados en temas sociales.

En el fondo, la impertinente pregunta acerca del papel desempeñado por la estructura se repite aquí de nuevo ya que estos autores, una vez analizado pormenorizadamente un grupo, se plantean en qué modo el grado de «embeddedness» influye en las relaciones sociales y limita la capacidad de acción. En opinión de Moody y White, el recurso a esta forma de trabajo permite huir tanto de las perspectivas que maximizan la libertad de la persona en sociedad como de aquellas otras que la reducen hasta prácticamente anularla. En este sentido, formulan una ligera crítica a la Network Exchange Theory, recuérdese, a la fusión de la teoría del intercambio y de la teoría de redes, la cual suele dar por sentada de modo erróneo una desigualdad en la distribución interna de poder que coloca a unos siempre por debajo de otros. Asimismo, proponen dinamizar la idea de red ya que con demasiada facilidad se asimila a fotos fijas invariables en las que el cambio histórico no ha lugar. Por último, y este sea quizás el punto en el que manifiestan los autores un mayor empeño, cada red debe ser puesta en relación con el resto de redes con las que convive, no a través de meros puntos de conexión sino como «muñecas rusas», es decir, solapadas, compartiendo miembros que desempeñan distintos roles en cada una de ellas, entrelazadas en una continuidad de vínculos humanos, etc. Un modelo de referencia, pues, que los historiadores deberían también asimilar, sobre todo para mejor completar propuestas que entre ellos han cosechado bastante éxito en los últimos años. Veamos.

Aun reconociendo que el término «acción» resulta en buena medida extraño al vocabulario de los historiadores, no ocurre así con otro cuasi sinónimo, no menos controvertido, como es el de «estrategia». A nuestro juicio, lo polémico de este concepto, de uso corriente sobre todo entre los estudiosos de la familia, fue puesto ya de relieve por Anderson en 1985. «El error conceptual aquí implícito es unir bajo el rótulo de acción aquellas acciones que son de hecho voliciones conscientes a nivel personal o local, pero cuya incidencia social es profundamente involuntaria (por ejemplo, la relación de la edad del matrimonio con el crecimiento de la población) con aquellas acciones que son voliciones conscientes a nivel de su propia incidencia social»55. Sin embargo, no suele citarse a este historiador como teórico del

${ }^{55}$ Anderson, P.: Teoría, política e historia. Un debate con E. P. Thompson. Madrid, Siglo XXI, 1985, p. 23. En otros ámbitos de las ciencias sociales se está también de acuerdo con esta distinción: «[...] ni la acción es reducible a su sentido subjetivo, ni las instituciones son lo que de ellas se piensa, pues hay 
concepto de estrategia, honor éste que se atribuye al antropólogo Barth ${ }^{56}$. Subrayó precisamente un tema ya tratado por nosotros: será el intercambio entre individuos o, en sus propias palabras, la transacción, lo que marque la pauta de las estrategias llevadas a cabo en una sociedad determinada ${ }^{57}$. Tras él, otros muchos siguieron la senda abierta. Baste citar a Bourdieu ${ }^{58}$ (el cual creía ver en la estrategia no un acto racional, sino más bien inducido), Davies ${ }^{59}$ o Tilly, cuya opinión reservamos para más adelante.

Pero antes de proseguir, a grandes rasgos, podría definirse la estrategia como una fórmula que engloba secuencias de decisiones tomadas por un actor que persigue unas metas, bajo un conjunto de reglas o constricciones, intentando anticipar consecuencias futuras y teniendo en cuenta las acciones y reacciones de otras personas ${ }^{60}$. El gran logro de esta opción interpretativa consistió en rescatar al individuo, también a la familia, del marco opresivo de la estructura, ahora bien, como señalaba acertadamente Anderson y otros con él, para poder elegir ha de haber primero una gama abierta de opciones y total libertad para escoger una de ellas.

Este matiz no ha escapado a los historiadores recientes, de ahí que haya tenido que reformarse la teoría para dar cabida, una vez más, a los condicionantes globales propios de todo tiempo, en otras palabras, mal que pese a los anti-estructuralistas, se ha reconocido la necesidad de vincular a las familias objeto de estudio con el resto de la comunidad y con las constricciones emanadas de ésta. También se ha intentado perfeccionar el esquema de trabajo mediante la indagación de las estrategias puestas en práctica por cada uno de los miembros de la familia, así como de los cambios en las mismas experimentados por los individuos a

siempre algo más, la lógica inconsciente de la sociedad que constantemente produce consecuencias, no queridas quizá, incluso no deseadas, pero que tienen que ser aceptadas como hechos que no derivan de nadie, hechos sin actor que los produce, hechos sociales propiamente dichos» (LAMO DE ESPINOSA, E.: «El objeto ...», p. 24).

56 BARTh, F.: Process and form in social life. Londres, Boston y Henley, Routledge \& Kegan Paul, 1981. Véase también VIAZZO, P. P. y LYNCH, K. A.: «Anthropology, family history, and the concept of strategy» International Review of Social History, 47:3, (diciembre 2002), pp. 423-452, p. 425. Para una aproximación centrada desde el punto de vista del antropólogo veánse las páginas 433-441 y también KERTZER, D. I.: «Anthropology and family history» Journal of Family History, (otoño 1984), pp. 201-216.

57 Al respecto matiza T. Engelen: «una estrategia, por definición, implica una elección entre distintas opciones. Cuando no hay opciones alternativas excepto el comportamiento que de hecho hallamos, es difícil hablar de estrategia» (ENGELEN, T.: «Labour strategies of families» International Review of Social History, 47:3, (diciembre 2002), pp. 453-464, p. 461; traducción propia).

58 Bourdieu, P.: El sentido práctico. Madrid, Taurus, 1991.

59 DAVIES, N. Z.: «Ghosts, kin, and progeny: Some features of family life in early modern France» Daedalus, 196, (1977), pp. 87-114.

60 FonTAINE, L. y SCHLUMBOHM, J.: «Household strategies for survival: An introduction» International Review of Social History, 47:3, (diciembre 2002), pp. 1-18, p. 6. Otra posible definición es la ofrecida por P. Moen y E. Wethington: «Estrategia adaptativa de familia es una construcción con un cierto atractivo para la intuición, explicando la familia como un participante activo en la sociedad global, un actor que ofrece respuestas, reelabora o reestructura constricciones externas y oportunidades» (MOEN, P. y WETHINGTON, E.: «The concept of family adaptive strategies» Annual Review of Sociology, 18, (1992), pp. 233251, p. 234, traducción propia). 
lo largo de su vida, introduciendo de esta forma una nueva perspectiva más dinámica ${ }^{61}$.

Tilly se preocupó, así, por buscar el vínculo entre las vidas personales y los comportamientos colectivos ${ }^{62}$ y supo detectar los inconvenientes que entraña el término estrategia. «Los historiadores emplean las estrategias familiares de dos formas: una que asume el hecho de que las estrategias realmente existieron en las mentes y en las acciones de los actores históricos y otra en la que las estrategias existen solamente en las mentes del analista» ${ }^{63}$. La advertencia no es gratuita, sobre todo si la empleamos para calibrar en su justa medida los importantes logros que ha traído consigo el estudio pormenorizado de las familias con el fin de conocer su trascendencia en procesos hasta hace poco considerados estructurales, como pudiera ser la revolución industrial ${ }^{64}$. Sin embargo, críticas más contundentes, no hablamos ya de «matices», ha recibido en los últimos años la opción que nos ocupa.

En primer lugar, se ha sobredimensionado quizá la racionalidad que se ha creído ver detrás de las estrategias ${ }^{65}$. En el caso de las familias, se les ha otorgado a menudo un poder sobre su devenir y una capacidad de autodefinición a lo largo del tiempo que, simplemente, adolece de una pérdida de perspectiva histórica. Engelen encuentra desproporcionada la libertad que se le ha reconocido al individuo a la hora de llevar a cabo sus acciones ${ }^{66}$. A Woolf, por su parte, lo que le llama la atención es el estrecho lugar que dejan este tipo de teorías para el afecto y los sentimientos, siendo un frío cálculo obsesionado por los ritmos económicos el director indiscutible del devenir social ${ }^{67}$. Por ello conviene estudiar "cómo de estratégicas son las estrategias». Siguiendo con el mismo símil, otros autores no se resisten a lanzar esta pregunta: «¿cómo de solidarias son las estrategias?», porque lo cierto

61 Moen, P. y Wethington, E.: «The concept ...», p. 246. Véase también el trabajo pionero de BERKNER, L. K.: «The stem-family and the developmental cycle of the peasant household: An eighteenth-century Austrian example» American Historical Review, 77, (1972), pp. 398-418.

62 «Tilly definió estrategia familiar adaptativa como un conjunto de reglas implícitas que guían el comportamiento de los miembros de la familia, de las familias en sí y de los hogares. Estas estrategias tienen dos funciones: la primera, ser un medio de cálculo familiar racional para tomar decisiones económicas y sociales que afectan a la familia en su conjunto; la segunda, una materialización de percepciones preexistentes y de prácticas centradas en el día a día, es decir, un conjunto de reglas implícitas útiles en la toma de decisiones para hallar soluciones a los problemas» (MOEN, P. y WETHINGTON, E.: «The concept ...», p. 235, traducción propia). Para una lectura directa, véase Tilly, L. A.: «Individual lives and family strategies in the French proletariat» Journal of Family History, 4, (1979), pp. 137-152.

63 Moen, P. y Wethington, E.: «The concept ...», p. 432 (traducción propia).

64 BAUD, M. y ENGELEN, T.: «Introduction: Structure or strategy? Essays on family demography, and labour from the Dutch N. W. Posthumus Institute» The History of the Family. An International Quarterly, 2:4, (1997), pp. 345-352, pp. 348-349.

65 Moen, P. y Wethington, E.: «The concept ...», p. 244.

66 ENGELEN, T.: «Labour ...», p. 456.

67 WoOLF, S.: «Teorías macro y microeconómicas y estrategias familiares: algunas reflexiones ingenuas y escépticas» Boletín de la Asociación de Demografía Histórica, XII:2/3, (1994), pp. 32-47. De igual modo T. Hareven y M. Baud desconfían del cierto determinismo económico que se deriva de algunos trabajos y abogan por la inclusión de valores culturales como factores influyentes. Véase respectivamente ENGELEN, T.: «Labour ...», p. 237 y BAUD, M. y ENGELEN, T.: «Introduction ...», p. 350. 
es que se sugiere siempre que intercambios, redes, estrategias,... llevan implícita una carga de solidaridad 68 .

Como puede comprobarse con estas breves notas, la aparición del concepto en cuestión fue muy bien recibida, renovó manidas formas de afrontar las incógnitas sociales, pero no por ello deja de ser susceptible de mejora. Cada vez se insiste más en que la perspectiva histórica dentro de cada grupo familiar debe ser contemplada ${ }^{69}$ con el objeto de no reificar los conceptos que sirven de apoyo a los argumentos, desvirtuando su sentido originario. Tampoco parece útil dotar a la sociedad de una especie de inteligencia propia, error éste que se comete a menudo, por ejemplo, al explicar las constantes demográficas observadas a través de la estadística como corroboración de determinadas estrategias ${ }^{70}$.

Algunos autores que venimos siguiendo sentenciaron en un reciente número de la International Review of Social History que el término «táctica» sería más adecuado para captar acciones de corto plazo sobre las que influyen factores externos ajenos a la voluntad de los individuos ${ }^{71}$. Más escéptico aún, Engelen no niega que existan estrategias, pero sí veta al historiador capacidad investigadora suficiente como para descubrirlas ${ }^{72}$. Por su parte, Moen y Wethington se decantan por la alternativa, ya sugerida por nosotros, del life course approach consistente en el estudio de las posiciones que el individuo va ocupando a lo largo de su vida ${ }^{73}$, las cuales, según los propios autores, implican una respuesta de las familias a sucesivas barreras estructurales ${ }^{74}$.

Queríamos concluir con estos autores, de nuevo, por haber hallado en ellos una opción intermedia, pero no debemos tampoco dejar de aludir al optimismo de quienes, a pesar de reconocer la dificultad de aplicar a la investigación el concepto de estrategia, confían en las técnicas del historiador para inferir qué empujó a las familias a actuar de una determinada forma y no de otra. Hay que diferenciar el corto y el medio plazo, advierten, para no incurrir en muchos de los errores aquí expuestos. La tradición asimismo puede coincidir con las estrategias, de hecho, una elección (racional casi siempre) que se muestre exitosa será adoptada de ma-

68 Moen, de acuerdo con T. Hareven, recuerda que lo que es funcional para una familia tal vez no lo sea para otra vecina (MoEn, P. y Wethington, E.: «The concept ...», p. 242 y BAUD, M. y ENGELEN, T.: «Introduction ...», p. 350). Para una visión global de la conflictividad que tiene en cuenta los postulados de historiadores y sociólogos, véase GARCíA SÁNCHEZ, M. A.: «La conflictividad social en la Europa moderna. Apuntes teóricos», Sociedad y utopía. Revista de Ciencias Sociales, 21, (mayo 2003), pp. 15-28.

69 VIAZZO, P. P. y LYNCH, K. A.: «Anthropology ...», p. 429 y Woolf, S.: «Teorías ...»

70 VIAZZO, P. P. y LYNCH, K. A.: «Anthropology ...», p. 440.

71 VIAZZO, P. P. y LYNCH, K. A.: Ibidem, pp. 450-451.

72 ENGELEN, T.: «Labour ...», p. 463.

73 KOK, J.: «The challenge of strategy: A comment» International Review of Social History, 47:3, (diciembre 2002), pp. 465-485, p. 473.

74 Moen, P. y Wethington, E.: «The concept ...», pp. 233. «Los modelos life-course combinan aspectos relativos a las teorías de elección racional y estructural dentro de un marco temporal con el objeto de ubicar la familia y las estrategias individuales de adaptación en un más amplio contexto histórico, social y cultural de oportunidades cambiantes y constricciones, recursos y demandas, normas y expectativas» (Moen, P. y Wethington, E.: «The concept ...», p. 245, traducción propia). 
nera general y entonces dejará entreverse en los datos globales que tengamos de una sociedad.

«En mi opinión, no es muy útil distinguir entre adaptación racional por un lado y estrategias innovadoras por otro. La innovación del comportamiento, la raíz del cambio social, parece ser un proceso gradual en el cual la gente, partiendo de sus propias experiencias, costumbres y normas, percibe que una solución particular puesta en práctica por otros - a menudo sus vecinos- es más efectiva que la suya» ${ }^{75}$.

Por fin, ha de ser la investigación empírica la que, a la luz de estas reflexiones, nos indique cuál de las opciones, dependiendo del objeto de estudio concreto, se muestra más certera. En ningún momento pretendimos desligar las teorías del plano factual sobre el que descansan los trabajos de historiadores y sociólogos, si bien el fin perseguido en estas páginas consistía ante todo en presentar un elenco bibliográfico debidamente contextualizado dentro de una larga tradición interpretativa.

\section{CONCLUSIONES}

Comenzábamos diciendo que las referencias al sujeto y la acción pueblan muchos de los trabajos escritos por los científicos sociales y por esta razón no sobran reflexiones en torno a este tema. Alivia saber que el arduo interrogante en el que nos hemos ocupado es calificado por un autor de la talla de Giddens como dilema número 1 de la sociología. «Lo más probable es que esta polémica no se resuelva nunca - afirma-, ya que ha existido desde que los pensadores modernos trataron de explicar sistemáticamente el comportamiento humano. Además, es un debate que no se restringe a la sociología sino que preocupa a los expertos de todas las ciencias sociales» 76 .

En cualquier caso, hemos de decir, a modo de balance final, que tal y como se refleja en el primer epígrafe la acción se erige en el origen indiscutible de cualquier formación social y es a partir de ella desde donde se derivan las normas que coordinan la convivencia de los individuos. A menudo los historiadores obvian este hecho, así como la renovación continua que experimentan esas reglas globales mediante la interacción de las personas.

En esta misma línea, del segundo apartado extraemos como conclusión que el profesional de la historia, a pesar de las trabas que le presenta su objeto de estudio, sí es capaz de analizar los comportamientos del sujeto en el pasado. De hecho, la aproximación a la estructura debe partir de abajo, debe ser una inferencia desde las observaciones individuales. Giddens ha sido quizás el que nos haya

75 KoK, J.: «The challenge ...», p. 472 (traducción propia).

76 Giddens, A.: Sociología. Madrid, Alianza, 2001, p. 717. 
mostrado más a las claras en qué consiste ese diálogo entre el sujeto y la estructura, constituyendo así la alusión a su obra una deriva en nuestro discurso hacia interpretaciones culturalistas. Ahora bien, los planteamientos recién referidos han dado lugar a menudo a una reificación de la cultura nada beneficiosa para los postulados metodológicos aquí defendidos. Por tanto, y para sortear este peligro, el recurrir al apoyo del grupo como paso intermedio ha sido entendido por nosotros como la garantía de un análisis más comedido. El estudio pormenorizado del grupo e integrado con las nociones de sujeto y estructura, lejos de invitar a la creación de compartimentos estanco entre estos estadios de análisis, nos ha conducido en el epígrafe cuarto a recapitular una serie de teorías que proporcionan un fundamento sólido para entender el funcionamiento interno de los colectivos.

Creemos haber podido conjugar de esta forma perspectivas y haber apuntado un beneficioso intercambio de ideas entre distintos campos de las ciencias sociales. Por añadido, la reflexión en torno a estas cuestiones permite afrontar mejor trabajos empíricos, los cuales, desgraciadamente, a menudo adolecen en nuestro país de cualquier atisbo de respaldo teórico. 